\title{
Improving Cognitive Functions of Dyslexics using Multi-sensory Learning and EEG Neurofeedback
}

\author{
Günet Eroğlu \\ Faculty of Engineering and Natural Sciences \\ Sabancı University, İstanbul, Turkey \\ gunet.eroglu@healthmobilesoftware.com
}

\author{
Serap Aydin \\ Dept of Biomedical Eng, Faculty of Engineering \\ University of Beykent, Maslak, İstanbul, Turkey \\ drserapaydin@hotmail.com
}

\author{
Müjdat Çetin \\ Faculty of Engineering and Natural Sciences \\ Sabancı University, İstanbul, Turkey \\ Dept. of Electrical and Computer Eng., University of Rochester, Rochester, NY, USA \\ mcetin@sabanciuniv.edu
}

\author{
Selim Balcisoy \\ Faculty of Engineering and Natural Sciences \\ Sabancı University, İstanbul, Turkey \\ balcisoy@sabanciuniv.edu
}

\begin{abstract}
AutoTrainBrain is a neurofeedback and multisensory based mobile phone software application, designed in Sabancı University laboratory with the aim of improving the cognitive functions of dyslexic children. It reads electroencephalography (EEG) signals from 14 channels of eMotiv EPOC+ and processes these signals to provide neurofeedback to child for improving the brain signals with visual and auditory cues in real time. AutoTrainBrain software has been applied to a 14year old dyslexic child, 10 minutes per week for 9 consecutive weeks.The EEG data has been analyzed by using the following three approaches: estimation of single-channel EEG complexity levels (entropy), spectral brain connectivity between two-channels (coherence), single channel relative Alpha band power ratio. Our experimental analysis shows that the proposed brain training system offers improvements based on the measures used in the three approaches mentioned above. This suggests such training may help increase the number of active cortical neurons and improve regional brain connectivity.
\end{abstract}

Keywords-Neurofeedback, EEG signal processing, multi sensory learning, dyslexia.

\section{INTRODUCTION}

Dyslexia is a specific learning disability that is neurobiological in origin. It has a prevalence of 10 to $15 \%$ of children. It is characterized by difficulties with accurate and/or fluent word recognition and by poor spelling and decoding abilities. These difficulties typically result from a deficit in the phonological component of language that is often unexpected given the otherwise normal cognitive abilities of the person as well as the provision of effective classroom instruction. Secondary consequences may include problems in reading comprehension and reduced reading experience that can impede growth of vocabulary and background knowledge [1]. Dyslexia is probably the most common neurobiological

978-1-5386-1501-0/18/\$31.00 (C) 2018 IEEE disorder affecting children. Reading problems manifest themselves mainly in the following areas: difficulty in learning to utilize correspondence regularities between graphemes and phonemes poor phonological awareness, i.e., awareness of the sound structure of words, especially phonemic awareness as manifested in the ability to analyze and manipulate sounds within a syllable and poor use of orthographic word reading strategies; and consequently inaccurate and non-fluent word identification. As a result of these difficulties, full alphabetic or phonological reading skills are often not attained [2]. The most frequently reported EEG abnormality in children with learning disabilities is increased theta relative power in their electroencephalography (EEG) signals relative to their peers. Several studies have reported higher amounts of delta and theta, and lower amounts of alpha in children with learning disabilities compared to normal children of the same age. An important percentage of children with learning disabilities show EEG patterns that are more typical for younger children, which has been interpreted as a delay of EEG maturation. Other groups of children with learning disabilities show paroxysmal EEG activity [3]. In neurofeedback applications, the user's brain activation is depicted in real-time with the goal of helping the user to gain control over specific aspects of the activity in his/her central nervous system. Hence, the user receives direct feedback about their actual brain activation pattern and consequently can learn to gain voluntary control over neural signals. By watching and listening to real-time multi-media representations of its own activity, the brain can modify its functionality and even its structure. Healthy, age appropriate brain activity is rewarded with visual, auditory or even tactile stimulation. In contrast, undesirable patterns of brain activity are ignored or even penalized [4]-[7]. AutoTrainBrain is a patent pending software application specifically designed for dyslexic children. Within this software application, a system and method for improving reading ability and cognitive functions is proposed, the system relying on a distinctive protocol 
of multi-sensory learning and EEG neurofeedback. The EEG neurofeedback protocol is specifically designed for learning disabilities and the EEG neuroofeedback system integrated with multisensory learning provides a powerful and robust tool for improving reading ability. The EEG neurofeedback system is easily usable and does not require technical expertise (Figure 1 and Figure 2).

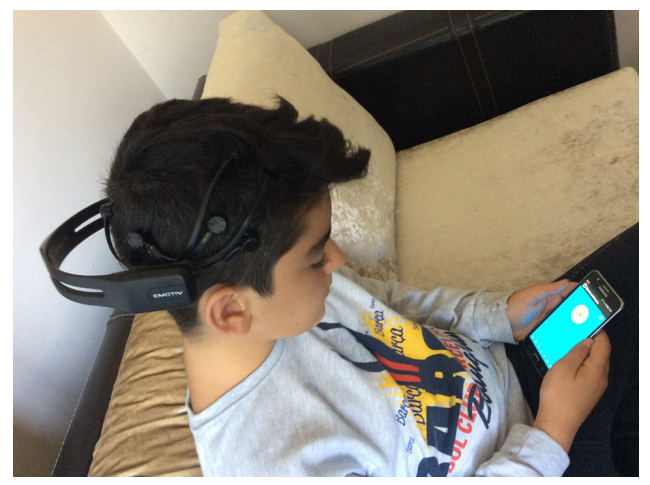

Figure 1: The usage of AutoTrainBrain

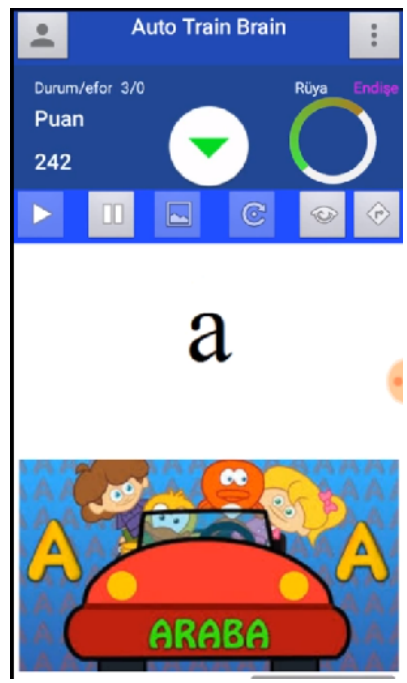

Figure 2: AutoTrainBrain Software User Interface

A single user software module/application on a mobile phone for improving reading abilities and cognitive functions in general is provided. Before the training or concurrently with the training, EEG signals are read from a sufficient number of electrodes (1-14) from the user's scalp and these EEG signals are translated to auditory and visual feedback to improve the user's brain performance.The software contains norm data collected from healthy people. This data is used for determining thresholds during neurofeedback to eliminate the side effects.

\section{MATERIALS \& METHODS}

\section{A. Subject and Experimental data}

A dyslexic child at the age of 14 voluntarily participated in this study. Before the experiment, his parents and him gave their informed consent after the experimental procedure was explained to them in accordance with guidelines set by the research ethics committee of Sabanci University. Throughout the experiments, eMotiv EPOC+ headset (http://www.emotiv.com) is used. Internal sampling rate in the headset is 2048 per secs per channel. This is filtered to remove main artefacts and alias frequencies then down-sampled to 128 per secs per channel.There are 14 EEG channels plus two references and 128 samples per second per channel is used. Electrodes were placed according to the 10-20 system. Before the experiments, the calibration of eMotiv Headset on the subject's scalps is done with MyEmotiv mobile application of eMotiv, each electrode is made sure to transfer EEG data with high quality.

\section{B. Study Design}

The main goal of the experiment is to reduce the slow brain waves, which is achieved by visual and auditory feedback provided online in real time by the Android Java program after processing the EEG data gathered from the subject's head. Before the experiment, the raw EEG signals in the resting, "eyes closed" state are measured for two minutes using eMotivPro software and the data are stored in .edf files. During the neurofeedback session, EEG data are recorded, and after the experiment, another two minutes of resting "eyes closed" state raw EEG signals are recorded. During these recordings, the subject is placed approximately 0.5 meters in front of a mobile phone and instructed to relax with eyes open. AutoTrainBrain software is written with Android Java and uses the Community SDK provided by eMotiv to communicate with eMotiv EPOC+ headset. Artefact removal and conversion from Analog to Digital is done by the standard eMotiv procedures. For 9 consecutive weeks, different sessions of neurofeedback by AutoTrainBrain are conducted for 10 minutes.

\section{Resting State Raw EEG processing}

For all analyses in this study, all of the 14-channel EEG data are recorded during the experiments in Theta (4-8 Hz), Alpha(8-12 Hz), Beta-1 (12-16 Hz), Beta-2(16$25 \mathrm{~Hz})$, Gamma $(25-45 \mathrm{~Hz})$ bands. As we have used libEDK.IEE_GetAverageBandPowers routine, we have collected averaged and cleaned data from eMotiv EPOC+ headset and used the session average band powers in our calculations.

\section{Raw EEG Data Analysis}

In several pieces of previous work, it has been shown that complexity/entropy, coherence and relative Alpha Power values indicate the wellness of brain [10], [11], [12]. The analysis is conducted by comparing the before Neurofeedback raw EEG resting state, eyes closed recordings with that of the after neurofeedback session and that of the consecutive sessions. The raw data are filtered by using a bandPass FIR filter $(1-50 \mathrm{~Hz})$. The artefacts are removed manually by using EEGLAB's data rejection options. The raw EEG signals collected from each electrode channel are segmented into 2second sliding windows. For each 2-second EEG segment, the spectral entropy and related Alpha Band $(8-13 \mathrm{~Hz})$ absolute power are calculated. As shown in Figure 3, 60 short segments are constructed for 2- minute recorded session. The EEG data has been analyzed by using the following three approaches: The EEG data has been analyzed by using the following three 
approaches: estimation of single-channel EEG complexity levels (entropy), spectral brain connectivity between two channels (coherence), single channel relative Alpha band power ratio. In these three methods, power spectral density of short EEG segment of 2-second has been commonly computed by using Burg Method based on AR model assumption where the best model order is estimated through ARfit algorithm, spectral brain connectivity between two channels, single channel relative Alpha band power ratio. In these three methods, power spectral density of short EEG segment of 2 seconds has been commonly computed by using Burg Method based on AR model assumption where the best model order is estimated through ARfit algorithm.

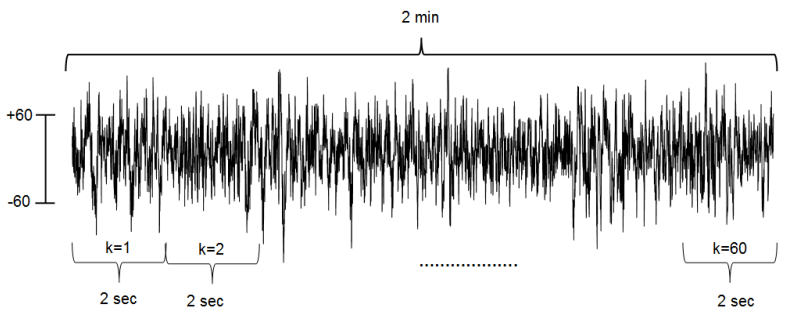

Figure 3: The raw EEG data segmentation

\section{RESULTS}

- If we compare the entropy which is estimation of single-channel EEG complexity levels (based on Burg method) calculated with EEG recordings before the first neurofeedback session with that after the first neurofeedback session, there are entropy increases in almost all channels (Figure 4).

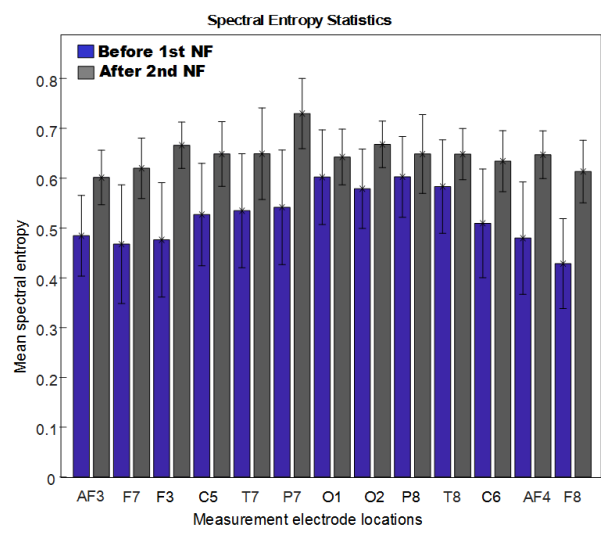

Figure 4: Spectral entropy based on Burg Method (Session 1)

- After the 9th neurofeedback session, the entropy which is estimation of single-channel EEG complexity levels (based on Burg method) increase compared with that at the first neurofeedback session is retained. In the 9th session, the difference between entropy increase before and after the neurofeedback is lowered. It means the cognitive improvement is permanent (Figure 5).

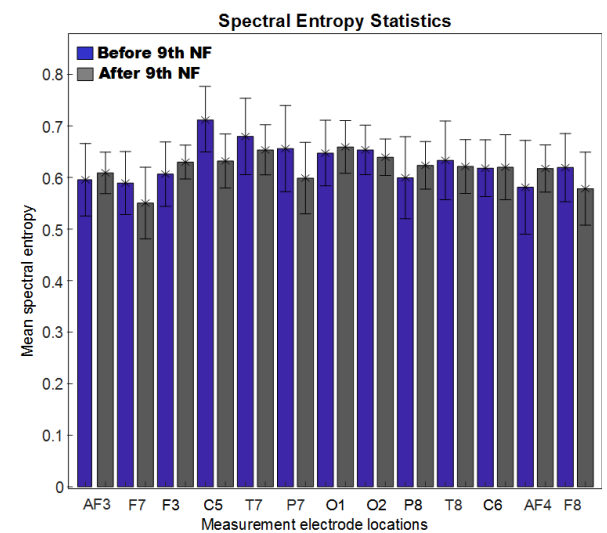

Figure 5: Spectral entropy based on Burg Method (Session 9)

- If we compare the Alpha Band Power $(8-13 \mathrm{~Hz})$ over total EEG $(0-30 \mathrm{~Hz})$ (Alpha Band Relative Power) calculated with EEG recordings before the first neurofeedback session with that after the first neurofeedback session, Alpha Band Relative Power is increased in almost all channels (Figure 6). After the 9th neuro-

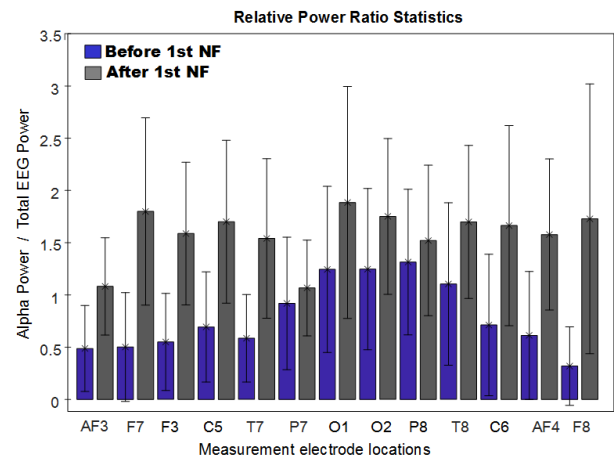

Figure 6: Single channel Alpha Relative Power increase (Session 1)

feedback session, the increase in Alpha Band Relative Power compared with that at the first neurofeedback session is retained. In the 9th session, the increase in Alpha Band Relative Power before and after neurofeedback is lowered. It means the cognitive improvement is permanent (Figure 7).

- If we compare the coherence calculated with EEG recordings before the first neurofeedback session with that after the first neurofeedback session, there are coherence increases in almost all channels (Figure 8). After the 9th Neurofeedback sessions, the increase in coherence which is spectral brain connectivity between two channels compared with that at the first neurofeedback session is retained. In the 9th session, the increase in cherence before and after neurofeedback is lowered. It means the cognitive improvement is permanent (Figure 9).

- The increase in entropy/ complexity, coherence, and Alpha Band Relative Power, observed more noticeably in the right hemisphere, may possibly be associated with cognitive performance enhancement mediated by 


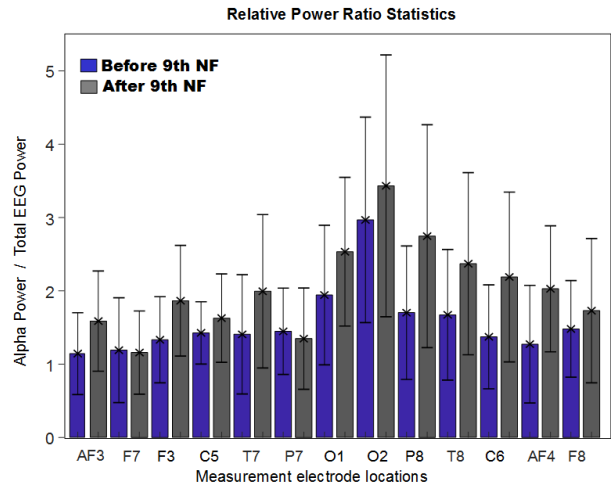

Figure 7: Single channel Alpha Relative Power increase (Session 9)

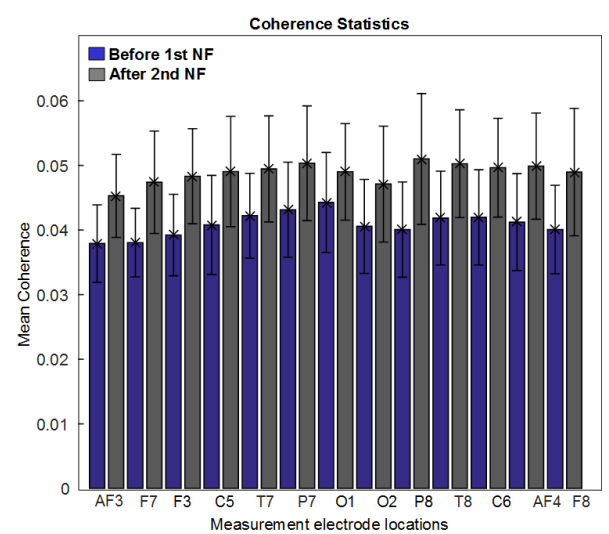

Figure 8: Increase in coherence (Session 1)

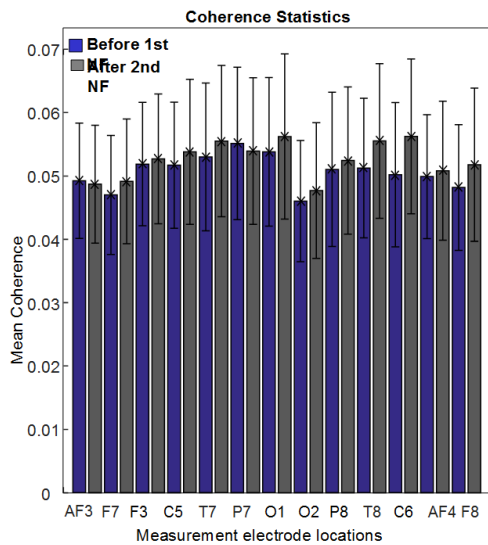

Figure 9: Increase in coherence (Session 9) neurofeedback sessions. Before neurofeedback session and after neurofeedback session, the increase is determined in the frontal lobes, which are mainly responsible for organization, managerial, and motor functions. Other increases are also found in Occipital and Parietal regions.

\section{REFERENCES}

[1] Lyon, G. Reid, Sally E. Shaywitz, and Bennett A. Shaywitz. "A definition of dyslexia." Annals of dyslexia 53.1 (2003): 1-14.

[2] Arns, Martijn, et al. "Different brain activation patterns in dyslexic children: evidence from EEG power and coherence patterns for the double-deficit theory of dyslexia." Journal of integrative neuroscience 6.01 (2007): 175-190.

[3] Fernandez, T., et al. "EEG and behavioral changes following neurofeedback treatment in learning disabled children." Clinical Electroencephalography 34.3 (2003): 145-152.

[4] Ninaus, Manuel, et al. "Neurofeedback and serious games." Connolly, TM, Hainey, T., Boyle, E., Baxter, G., Moreno-Ger, P.(eds.) (2013): 82110 .

[5] Walker, Jonathan E., and Charles A. Norman. "The neurophysiology of dyslexia: A selective review with implications for neurofeedback remediation and results of treatment in twelve consecutive patients." Journal of Neurotherapy 10.1 (2006): 45-55.

[6] Niv, Sharon. "Clinical efficacy and potential mechanisms of neurofeedback." Personality and Individual Differences 54.6 (2013): 676-686.

[7] Nazari, Mohammad Ali, et al. "The effectiveness of neurofeedback training on EEG coherence and neuropsychological functions in children with reading disability." Clinical EEG and neuroscience 43.4 (2012): 315-322.

[8] Marple, S. Lawrence, and S. Lawrence Marple. Digital spectral analysis: with applications. Vol. 5. Englewood Cliffs, NJ: Prentice-Hall, 1987.

[9] Burg, John Parker. "The relationship between maximum entropy spectra and maximum likelihood spectra." Geophysics 37.2 (1972): 375-376.

[10] Czigler, Balázs, et al. "Quantitative EEG in early Alzheimer's disease patients-power spectrum and complexity features." International Journal of Psychophysiology 68.1 (2008): 75-80.

[11] Sheppard II, William D., and Robert W. Boyer. "Pretrial EEG coherence as a predictor of semantic priming effects." Brain and language 39.1 (1990): 57-68.

[12] Rosso, Osvaldo A. "Entropy changes in brain function." International Journal of Psychophysiology 64.1 (2007): 75-80. 